\title{
Constrained and Unconstrained evolution of "LCR" low-pass filters with oscillating length representation
}

\author{
Yerbol Sapargaliyev, and Tatiana Kalganova
}

\begin{abstract}
The unconstrained evolution has already been applied in the past towards design of digital circuits, and extraordinary results have been obtained, including generation of circuits with smaller number of electronic components. In this paper both constrained and unconstrained evolutions, blended with oscillating length genotype sweeping strategy, are applied towards design of analogue "LCR" circuits. The comparison of both evolutions is made and the promising results are obtained. The new algorithm has produced the best results in terms of quality of the circuits evolved and evolutionary resources required. It differs from previous ones by its simplicity and represents one of the first attempts to apply Evolutionary Strategy towards the analogue circuit design. The obtained results are compared in details with lowpass filters previously designed.
\end{abstract}

\section{INTRODUCTION}

$\mathrm{T}_{\mathrm{p}}$ HE Evolvable Hardware (EHW) is one of the most promising areas of today's electronics. Evolutionary Algorithm (EA) applied towards reconfigurable hardware enables to find a solution among global solution space. The EHW where the ultimate goal is a circuit design is also referred as Evolutionary Electronics [1], [4]. The evolutionary electronics gives an alluring opportunity for an amateur in the field of Electronics to reach out the same results as professional one, possessing mostly the knowledge of Darwinian's laws and inspiration. The EA, navigated by a fitness values, provides randomly created and mutated chromosomes (Fig.1). Each chromosome encodes the structure for a circuit and has to be evaluated by a fitness function assigning a fitness value. The last one shows how close the current hardware structure by its behavioral and circuit characteristics to the required one. The circuits evolved may have unconventional designs and less of all depend on personal knowledge of a designer [1]-[11].

For instance, using simulation software (extrinsic EHW), low-pass filters [2]-[7] and amplifiers [3]-[5], [8] are successfully designed with the help of EA. Moreover, the structure and element parameters of reconfigurable hardware by itself could be set as an evolutionary target [10]. It seems quite promising and interesting perspective to evolve the reconfigurable chip oriented to the implementation of further evolutionary experiments. However, it implies the strict

Y. Sapargaliyev is with School of Engineering and Design, Brunel University, Uxbridge, Middlesex, UB8 3PH, UK (e-mail: yerbol.sapar@ brunel.ac.uk).

T. Kalganova is with School of Engineering and Design, Brunel University, Uxbridge, Middlesex, UB8 3PH, UK (e-mail: tatiana.kalganova@brunel.ac.uk). requirements towards the evolutionary technique, including: the sweeping strategies [3], the circuit growth strategy [2][11], the EA parameters [12], [13] and the circuit representation technique [2]-[11].

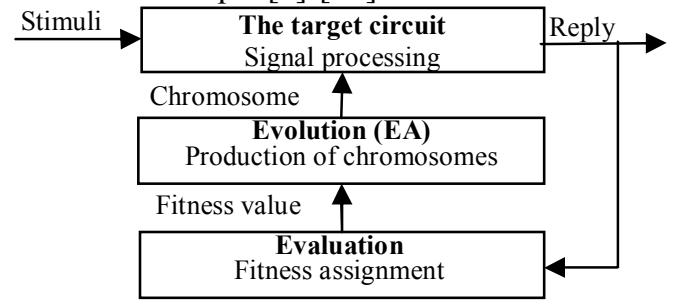

Fig.1. The concept of EHW. The target circuit here could be a real circuit with evolution process inside (intrinsic EHW), simulated by software with evolution outside of a real circuit (extrinsic EHW) or both of them (mixtrinsic EHW).

In [14] the unconstrained evolution, both spatially and temporally, has been applied intrinsically towards the digital FPGA-based reconfigurable hardware. By releasing the full repertoire of behaviors that FPGA can manifest, namely, allowing any connections among modules, letting the evolution to evolve the granularity of modules as well as the regimes of synchronization, evolution has been able to find a highly efficient electronic structure, which requires 1-2 orders less silicon area to achieve the same performance as conventional design does. Once fully unconstraining the design methodology rules, the natural behavior of analogue elements started to be exploited inside a circuit [14].

In analogy to this approach, the unconstrained evolution could be applied towards the originally analogue circuits. In this sense, the range of circuit-structure-checking rules at the netlist composition stage, prohibiting the invalid circuit graphs, are regarded as the main constraints for the design methodology.

In this paper, we consider both constrained and unconstrained extrinsic evolution for analogue "LCR" circuit design of low-pass filter. Absolutely agreeing with the factors set in [5] as favorite for evolutionary circuit design: E-12 series of component parameters and modesty in element amount within a circuit, we also set as important the simplicity of the evolutionary technique. Last one makes the experiments to be reconstructed and the same results easier to retain. We utilize the simplest oscillating length genotype (OLG) sweeping strategy [3] that together with unconstrained evolution produces much better results that previously achieved. The experimental results enabled us to compare performance of unconstrained evolution against constrained one as well as techniques developed previously. The description of an evolutionary technique is given in 
comparison with designs evolved in [2]-[5] and a filter designed conventionally in [15].

The paper is organized as fallows. The next section gives an overview of previous work in the area. Section III describes the problem to be evolved. Section IV introduces the both evolutionary techniques, constrained and unconstrained. Section V describes the experimental results. Section VI compares the obtained results with the filters evolved previously as well as designed conventionally. And, finally, the last section concludes the paper.

\section{PREVIOUS WORK}

The importance of analogue evolutionary circuit design is well described in [3]. Most of the works in the analogue circuit design start from evolving a passive low-pass filters, that is a convenient tool for probation of evolutionary technique and tuning the EA parameters towards the more sophisticated designs [2], [3], [10]. Low-pass filter circuits have deeply developed theory supported by numerous examples of real-world applications. Moreover, this task can be scaled along the level of difficultness by varying the steepness in the transition band and attenuation requirements.

The considerable results were obtained by Koza et al in [2]. They used Genetic Programming (GP) circuitconstructing program trees with four kinds of circuitconstructing functions. They also used automatically defined functions and enabled certain substructures to be reused. Last one let them to get as results filters with regular structures within the circuit. They have evolved three different low-pass filters with superior results. The main drawback of this experience is that the technique requires large computing power to adopt the same methodology as

\begin{tabular}{|c|c|c|c|c|c|c|c|c|c|}
\hline & $\begin{array}{c}\text { Koza } \\
\text { et al, [2] }\end{array}$ & $\begin{array}{c}\text { Lohn et al, } \\
{[3]}\end{array}$ & $\begin{array}{l}\text { Goh, } \\
\text { et al [5] }\end{array}$ & $\begin{array}{l}\text { Zebulum, } \\
\text { et al [4] }\end{array}$ & $\begin{array}{c}\text { Grimbleby } \\
{[7]}\end{array}$ & $\begin{array}{l}\text { Dastidar, } \\
\text { et al [8] }\end{array}$ & $\begin{array}{l}\text { Ando, } \\
\text { et al [6] }\end{array}$ & $\begin{array}{l}\text { Sripramong } \\
\text { et al [9] }\end{array}$ & 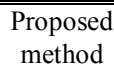 \\
\hline Publication year & 1997 & 2000 & 2000 & 1998 & 1999 & 2005 & 2003 & 2002 & 2006 \\
\hline Type of circuit evolved & $\begin{array}{c}\text { LC, } \\
\text { LCR, } \\
\text { LCRMD }\end{array}$ & $\begin{array}{l}\text { LCR, } \\
\text { MR }\end{array}$ & LCR & LCR, MR & $\mathrm{LC}$ & M & LCR & LCRM & LCR \\
\hline Type of EA & GP & GA & GA & GP,GA & GA & GA & GP,GA & $\begin{array}{l}\text { ES+sim. } \\
\text { annealing }\end{array}$ & ES \\
\hline $\begin{array}{l}\text { Circuit-structure-checking } \\
\text { rules }\end{array}$ & Partially & Yes & Yes & Yes & N/A & Yes & Yes & Yes & No \\
\hline Parameter optimization & No & No & No & No & $\begin{array}{c}\text { Yes: } \\
\text { numerical }\end{array}$ & Yes: GA & $\begin{array}{c}\text { Yes: } \\
\text { GP,GA }\end{array}$ & $\begin{array}{l}\text { Yes: hill- } \\
\text { climbing }\end{array}$ & No \\
\hline Sweeping strategy & ILG & ILG & ILG & $\begin{array}{l}\text { ILG,OLG, } \\
\text { UDIP }\end{array}$ & ILG & OLG & N/A & Fixed & OLG \\
\hline Circuit growth method* & 1 & 2 & 1 & 1 & 1 & 1 & 1 & No & 1 \\
\hline
\end{tabular}

* 1- technique where the place for a new element within a circuit is to be chosen arbitrarily, 2- where the circuit growth is along the way the current/voltage usually goes from input to output. N/A - data is not available. L-inductor, C-capacitor, R-resistor, M-transistor, D-diode. well as the methodology by itself is very complex for implementation.

The larger computational efforts in a circuit evolution required by GP were proven by works of Zebulum et al [4] and Ando et al [6], where they have given the comparison between GP and Genetic Algorithm (GA). The first work was made as analogy to biology concept with comparison of performance among different types of variable length chromosome strategies, while in the second one there was intrinsic evolution of real hardware for robustness purposes.

As well as in any evolutionary search, in electronic circuit design the freedom of evolutionary search is respected as crucially important for successful results. In [2], [3], [5], [8], [9] the freedom of search is emphasized, but has not been realized completely. The circuit-structure-checking rules prevent the invalid circuit graphs to be sent to evaluation or even to be generated. In [2] there has been a procedure providing the DC path to ground from each node of a circuit by adding the giga-Om resistance, allowing any kind of connections among capacitors. This is letting to avoid the most of "node floating" errors. But, they have not applied the similar approach to avoid the errors caused by "loops involving AC source or inductors". However this has enabled them to reach the amount of invalid circuit graphs up to $2 \%$. Later, they simplify each circuit by removing these redundant resistances and replacing all series and parallel compositions. 
Circuit growth methodology refers to the way in which the new element is added to a growing circuit. We distinguish 2 kinds of circuit growth techniques: Lohn's [3], where a new element is placed along the way the current usually goes from input to output, and the technique where the place for a new element is chosen randomly, used by mostly all other researchers [2]-[9]. First approach is good for avoiding invalid circuit graphs, thus reducing the search space. The second approach is very important for the work, considered in this paper, since it gives more freedom during evolutionary process.

According to [3], sweeping strategies refer to the way in which the different dimensionalities of the genome space are sampled by the EA. There are three kinds of sweeping strategies introduced by Zebulum et al in [4]: Increasing Length Genotypes (ILG), Oscillating Length Genotypes (OLG) and Uniformly Distributed Initial Population (UDIP). ILG and OLG strategies have shown superior results for analogue circuit design.

The previous development in evolution of analogue circuits design is summarized in the Table 1 . The analysis of Table 1 reveals that all the approaches developed in analogue circuit domain previously are based on the circuitstructure-checking rules for avoiding the invalid circuit graphs. In contrast, the experimental results were promising for evolution of digital circuits in the unconstrained search space [14]. Thus, this work aims on implementation and comparison of performance of constrained and unconstrained evolutions in analogue domain. Furthermore, the approaches introduced previously for analogue circuit design provided search with GP and GA, and none of them used purely ES.

Also, the majority of algorithms for low-pass filter design used the ILG strategy as a sweeping strategy; however in the work of Zebulum et al [4] the OLG has shown excellent results for analogue circuit design, and the best for the lowpass filter.

\section{PRoblem STATEMENT}

A low-pass filter passes low frequencies fairly well, but attenuates high frequencies. An ideal low-pass filter completely eliminates all frequencies above the cut-off frequency while passing those below unchanged. The transition region is infinitesimal. It can be realized mathematically by multiplying with the rectangular function in the frequency domain or, equivalently, convolution with a sinc function in the time domain.

However, the ideal filter is not realizable for real signals because the sinc function extends to infinity. The filter would therefore need to predict the future and have infinite knowledge of the past in order to perform the convolution. Real filters for real-time applications approximate the ideal filter by delaying the signal for a small period of time, allowing them to "see" a little bit into the future. Greater accuracy in approximation requires a longer delay. Two types of filters are shown on Fig.2: ideal with no transition band at all and the real one with transition band.

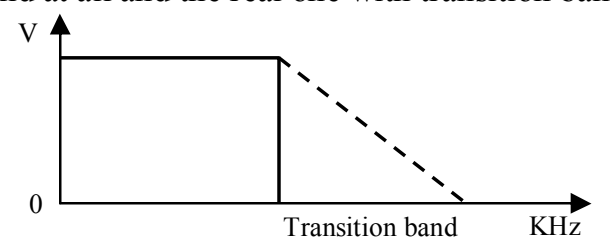

Fig.2. The circuit response of ideal and real low-pass filters. Last one is dashed and with transition band.

Previously the behavior of low-pass filters between frequencies $1 \mathrm{~Hz}$ and $100 \mathrm{KHz}$, cut-off frequency $1 \mathrm{KHz}$ and transition band $1 \mathrm{KHz}$ has been actively researched through in [2]-[3], [4]. Thus, the performance of proposed evolutionary technique could be evaluated more precise if the evolution target will have exactly the same filter properties. Moreover, the filters could be of two types "LC" and "LCR". The evolution of LC filters was considered in [2], [5], [7] and "LCR" filters were discussed in detail in [3], [4], [6], [7], [11]. In this paper we will attempt to evolve the "LCR" filters.

\section{CONSTRAINED AND UnCONSTRAINED EVOLUTION OF "LCR" CIRCUITS}

\section{A. Algorithm overview}

The structure of proposed system contains 4 main steps shown on Fig.3.

Step 1: The Start-block provides the start terms. At the start the circuits have to be preliminary grown up a little from the embryo-circuit in random manner. The Start-block also sets for the whole system such the terms as kind of circuits to evolve ("LCR"), seed for random number generator, the characteristics of filters to be evolved and the list of elements' parameters to be used by evolution. Last one is set to E-12 series of the totally 51 parameters for each element.

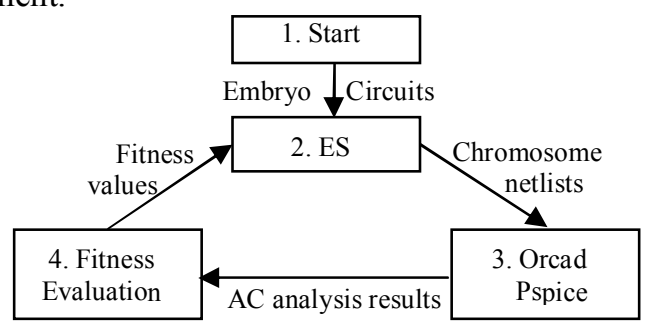

Fig.3. The flowchart of the experiment

Step 2: ES part sets the particular parameters of ES, such as: mutation rate, population size, selection criteria and termination terms. It modifies the genotype and produces the population of chromosomes in form of cir-batch-file towards OrCAD Pspice. At this stage the constrained evolution also includes the circuit-structure-checking rules, prohibiting invalid circuits to be generated. Unconstrained evolution here applies the special design rules that allow the circuit generation to be unconstrained.

Step 3: Pspice has been used in non-interactive batch simulation mode to reduce the evaluation time.

Step 4: Fitness evaluation block, as described bellow, 
fairly assigns the fitness values towards each circuit from $\mathrm{AC}$ analysis produced by Pspice in the form of batch out-file and passes the results to ES block. The PC program written in $\mathrm{C}$ programming language describes all 4 parts and unites them in one code. This stage also contains the circuit simplification procedure reducing the redundant elements and uniting the serial and parallel structures.

\section{B. The embryo-circuit and Evolutionary Strategy}

The embryo-circuit refers to the elements that are definitely known as essential for the target circuit, stay unchangeable during all the evolution and take place in each circuit netlist. In our case of "LCR" low pass filter as the target circuit, there are 3 such kind of elements: AC voltage source, source resistance and load resistance. We define the embryo circuit similar to the most popular case [2], [3], [5][7], [11], where the circuit is driven by an incoming AC voltage source with a $2 \mathrm{~V}$ amplitude, has the source resistance $R_{\text {source }}=1 \mathrm{k} \Omega$ and the load resistance $R_{\text {load }}=1 \mathrm{k} \Omega$ (Fig.4). The output voltage is measured on the pins of Rload.

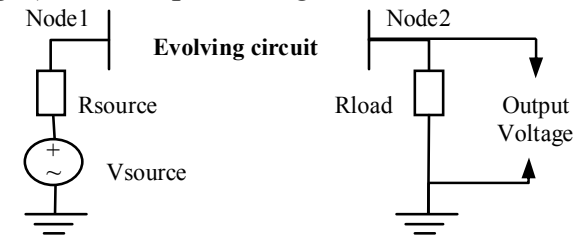

Fig.4. Embryo Circuit.

An example of Pspice netlist with correspondent schematic is shown on Fig. 5, where three embryo elements followed by the netlist are shown.

The circuit growth methodology is very simple and is similar to one reported in [5]: adding one gene to each chromosome at the same time.

The improvement of the circuits is driven by evolutionary strategy with disruptive selection scheme [12].

\section{Chromosome representation}

The linear circuit representation is proposed for use, similar to one that exploited in [4]. That is every element of a circuit is represented as a particular gene, and each of 4 element's features: name of an element, its parameter, and every its pin is represented by particular loci in a gene. The main advantage of this chromosome representation is that it directly maps the description of one element in the Pspice netlist. The number of genes in chromosome equals to the number of elements in a circuit. Fig.5 demonstrates the netlist of the circuit and its corresponding schematic. The circuit contains four elements (apart from embryo-elements). Therefore the chromosome representation contains four genes. Each gene is described by four loci. For instance, the first gene in the netlist is "L_0 12 1.8e-1", which describes the first inductor with pins $\mathrm{N} 1=1$ and $\mathrm{N} 2=2$ and with a value of $1.8 \mathrm{e}-1$ Henry. The first loci is reserved for the element's type ( $\mathrm{L}$ or $\mathrm{C}$ or $\mathrm{R}$ ), the second and the third loci are reserved for the first and the second pins, and the last loci is for the element's parameter. Similarly the remaining genes in the chromosome are encoded.

\section{Constrained evolution}

The usage of the circuit-structure-checking rules to prevent evaluation of invalid circuit graphs by simulation software was the only way before in analogue circuit domain to reduce the errors during circuit analysis. However, in this work we regard these rules as constrains and call such kind of evolution as constrained evolution. In [2] these constrains were partially released by adding to each node of a circuit the Giga-Ohm resistance, and the best results by circuit characteristics were obtained. The following subsection describes the main difference between constrained and unconstrained evolutions.

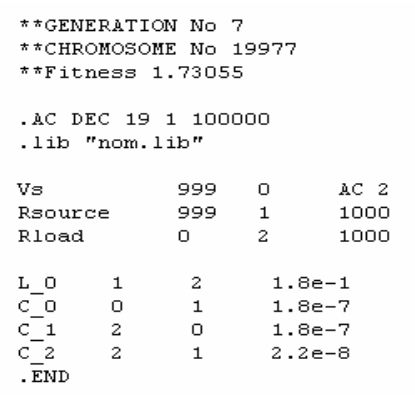

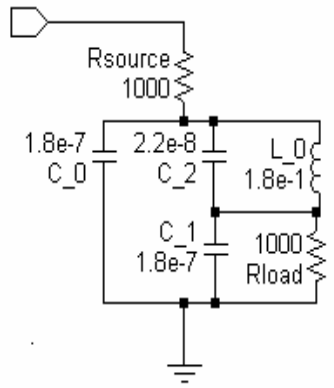

Fig.5. Chromosome representation at constrained evolution. The typical netlist and the correspondent schematic.

\section{E. The essence of unconstrained evolution in " $L C R$ " circuit design domain}

We call absolutely unconstrained evolution of an analogue circuit the process of a circuit netlist generation during which no any circuit-structure-checking rules applied and all the circuits are counted as valid graphs except ones that have elements with dangling nodes and with isolated subcircuits. There are two main kinds of invalidities that unacceptable for most of simulation software: the nodes that have no DC path to ground (tackled in [2]) and loops that involve inductors and/or voltage source. The most of methodologies in the area (Table 1) just prohibit such kinds of invalidities to appear. In the case of "LCR" circuits, the adding to each capacitor the Giga-Ohm resistance in parallel and the adding to each inductor the Micro-Ohm resistance in series, at the stage of Pspice cir-file generation, allows to avoid these invalidities. We call such kind of resistance as $R$ support. Using R-support and avoiding the dangling nodes makes almost any randomly generated circuit as valid, and indeed becomes an absolutely unconstrained.

Fig.6 demonstrates how unconstrained evolution generates the circuits with R-support. The circuit depicted on Fig. 5 once being processed by unconstrained evolution will have the view shown on Fig.6. Each element line describing inductor (L_0 on Fig.6) is followed by R-support element (Rl_1) in series with parameter 0.1 Micro Ohm; and each element line describing capacitor ( $\mathrm{C}_{-} \mathrm{No}$ ) is followed by $\mathrm{R}-$ support element (Rc_No) in parallel with parameter 1 Giga Ohm. 


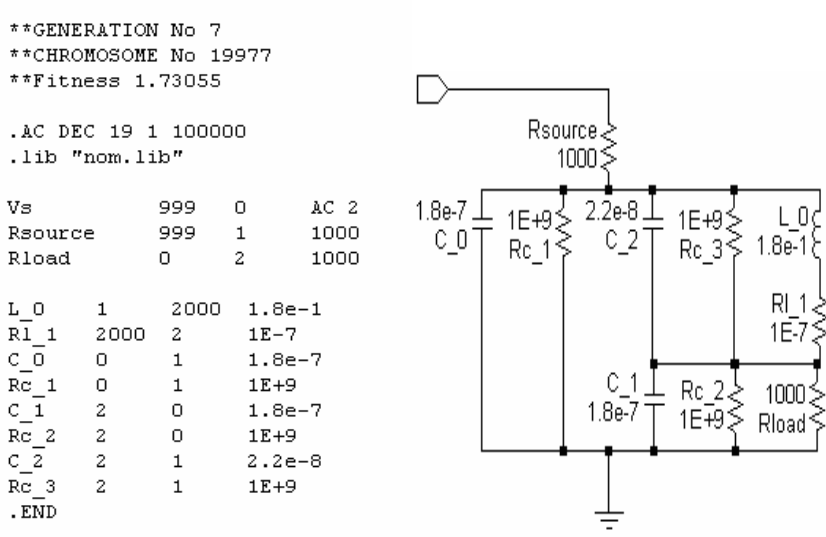

Fig.6. Chromosome representation at unconstrained evolution. The typical netlist and the correspondent schematic with R-support elements The three first elements (Vs, Rsource and Rload) compose the emryo-circuit.

If circuit-structure-checking rules are applied to a circuit after fitness assignment as a part of the simplification procedure, that is, every R-support element is checked whether it could be removed without damage to the current fitness of a circuit, then the circuit could contain a few Rsupport elements or even does not contain them at all. For instance, the circuit on the Fig.6 after simplification process becomes the circuit on the Fig. 5 .

\section{F. Mutation}

The whole process consists of three types of operations over the circuit. Every time the best fitness of a generation is not improved, the population falls into "Add new element mutation" (ANEM) procedure, i.e. one randomly generated gene is added to each chromosome except the chromosome with the best fitness value. At the start the number of nodes in the embryo circuit is equal to three: input, output and earth. The further growth circuit could increase the amount of nodes, if randomly generated node numbers for both pins are equal. In this case a new element splits the elements at the node and comes between them. ANEM procedure could be applied repeatedly, so the real difference in length between the best chromosome and others is oscillating and could reach numbers of genes.

However the "Delete element mutation" (DEM) will delete one gene if the difference between the shortest chromosome and the largest one in the generation greater than 2 genes. Thus the evolution can focus on processing chromosomes of three different neighboring sizes.

The "circuit structure mutation" (CSM) performs mutation over any of four loci of randomly chosen gene. If the mutation comes to a pin connection, the whole structure of a circuit is changed. However the total amount of elements stays unchangeable, the number of nodes of a circuit could be reduced or increased. The first case occurs if the pin changing its connection leaves alone the pin of another element, so the last one has no way as only to connect to the first (unchangeable) pin node of mutated element. In the second case, the total node number increases, if the mutated pin comes to the unchangeable pin of the same element, as mentioned above in ANEM case.
At the start the population comes to ANEM for preliminary circuit growth, and, after evaluation, it falls into CSM. The following switching rule between ANEM/DEM and CSM does manage: whenever the mutation within CSM does not bring any improvement in the fitness, the algorithm immediately switches to ANEM/DEM. As experiment show the fitness of an individual never improves by infinitesimal values, what makes the switch period between two kinds of mutations finite in time. For instance, having the fitness at the second generation 4.6492 and the best fitness 0.0040 at the 80th generation, we have set the value of $1 \mathrm{E}-5$ as the minimum fitness improvement to keep mutation within CSM. Whenever this improvement is less, all the population falls into ANEM/DEM. While CSM searches for the best circuit within the given amount of elements, it unavoidable brings to a fitness improvement stuck. But the adding one element to a circuit during ANEM stage significantly improves fitness reviving the whole process (Fig.7).

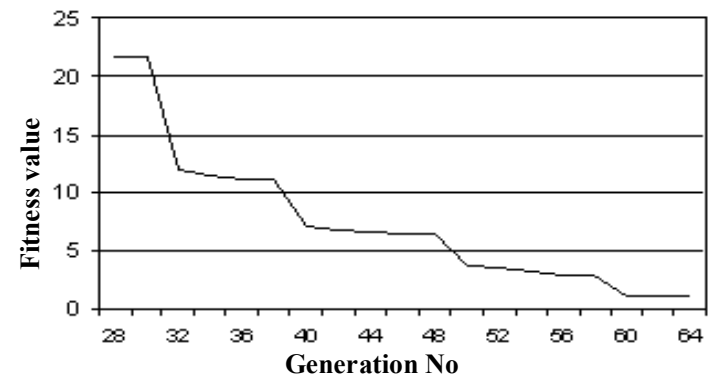

Fig.7. The fragment of the ordinary experimental data flow: dependency of the best fitness value along the generation number. At generations 29 , 38,48 and 58 the population of chromosomes has gone through ANEM stage, i.e. one element has been added to each circuit.

\section{G. Fitness Function}

We perform the AC-analysis along 96 points between 1 $\mathrm{Hz}$ and $100 \mathrm{kHz}$ (19 per decade), and measure the absolute deviation voltage between ideal value and produced by Pspice. We set the fitness evaluation in the analogy with [2], that is we distinguish as acceptable a voltage in the passband of between $970 \mathrm{mV}$ and $1 \mathrm{~V}$ (i.e., a passband ripple of 30 $\mathrm{mV}$ or less) and a voltage in the stop-band of between $0 \mathrm{~V}$ and $1 \mathrm{mV}$ (i.e., a stop-band ripple of $1 \mathrm{mV}$ or less):

$$
F_{1}=\sum_{i=0}^{P}\left|V_{\text {ideal }}^{i}-V_{\text {measured }}^{i}\right|,
$$

where $V_{\text {ideal }}^{i}$ is the voltage in $i$-th point for ideal filter and $V_{\text {measured }}^{i}$ is the voltage in the $i$-th point obtained for evolved filter; $p$ is a number of points evaluated in both stop and pass bands.

And we regard any voltage lower than $970 \mathrm{mV}$ in the passband and any voltage above $1 \mathrm{mV}$ in the stop-band as unacceptable, punishing it by multiplier 10: $F_{2}=10 \times F_{1}$.

The transition band is regarded as the "don't care" band where the fitness is supposed to be equal zero. In the case of $1 \mathrm{KHz}$ transition band it consists of five points between 1 $\mathrm{kHz}$ and $2 \mathrm{kHz}$. The error circuits are not analyzed by Pspice 
and assigned to the worst fitness value that never could be reached by other circuits.

\section{EXPERIMENTAL RESULTS}

\section{A. Initial settings}

The purpose of the experiments bellow is to compare constrained and unconstrained evolutions on the example of low-pass filter.

Population size of 20,000 chromosomes is set. Experimentally it has been established that the disruptive selection scheme [12] is the best: only $9 \%$ of the best chromosomes and 1\% worst ones are to be chosen for the next generation. Being chosen for the next generation each chromosome contributes $10 \%$ of the next population size. Static mutation rate of 5\% then applied to each chromosome randomly changing with equal probability every loci of a gene. ES is deserved the name of most simple EA among all, because it doesn't content the recombination stage: all the offspring chromosomes are identical to a correspondent parent.

The potential problem of the approach described is that without having enough diversity, the best fitness value could not growth at all within CSM stage and the evolution could just run quickly though out the ANEM stage, exceeding the element limit. There are two ways to tackle this problem: increase the size of the population and, keeping smaller population size, to increase the number of generations (repetitions) that population goes through CSM. For example, technically speaking, a population of 20,000 chromosomes equivalent to a population of 2,000 chromosomes with 10 repetitions or to a population of 50 with 400 repetitions. We used larger-population-size approach because it gives the advantage in speed: throughout all the three mentioned cases the average chromosome processing speed were 2100, 860 and 250 chromosomes per minute correspondently. It can be explained by the fact that OrCAD Pspice requires the Weblicensing each time (generation) the software runs, that is, 10-20 seconds required for each generation to be loaded independently of its size. Therefore in further stages we used only the ES with population size of $20,000^{1}$.

We set as a termination criteria reaching either of the following conditions: the number of circuit elements before simplification 28 (except R-support and embryo elements), the best fitness value $10^{-3}$, whole number of individuals evaluated (population_size $\times$ No_of_generations) $3 \mathrm{mln}$., and the time length of experiment 20 hours. According to experience, the most useful termination condition was element limit. The results presented bellow are the best out of 5 attempts for both experiments performed with 5 seeds

\footnotetext{
${ }^{1}$ Despite the good results have been received with this population size, there are no reasons why this size should not be increased. In fact, in here we were only driven by convenience of processing the Pspice out-file, which size in 20,000 population case reaches 210MB. We used PC Pentium-4, 3GHz, RAM 1GB.
}

for the random number generator.

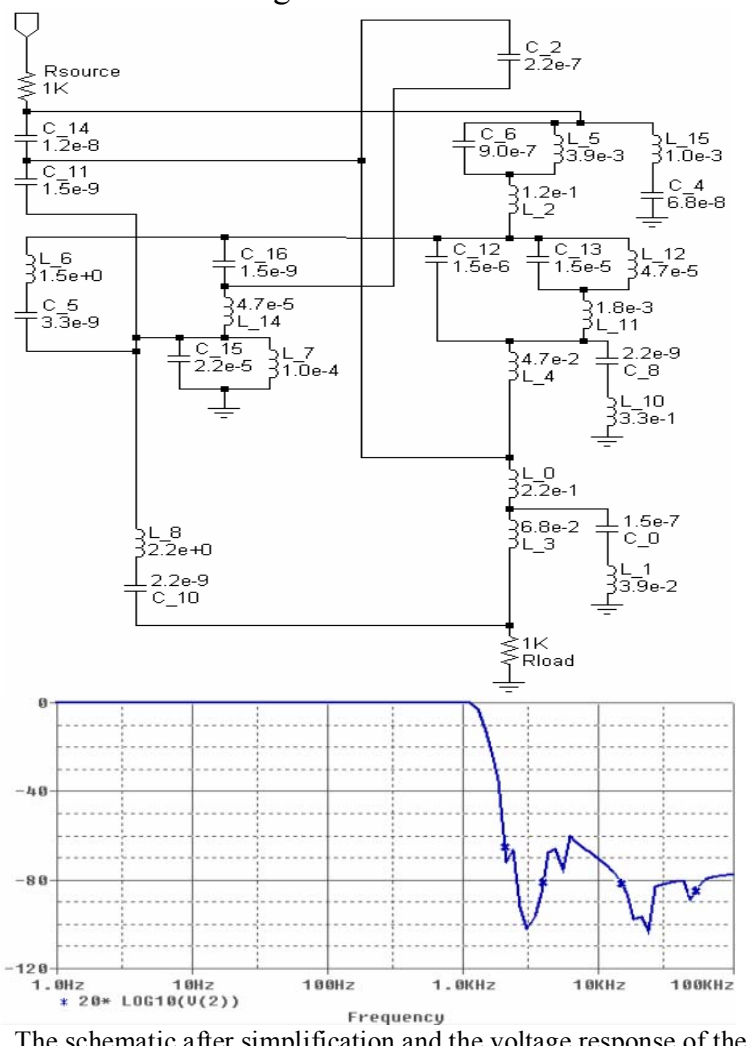

Fig.8. The schematic after simplification and the voltage response of the best low-pass filter evolved at Experiment A.

\section{B. Experiment A: Constrained evolution}

The purpose of Experiment A is to evolve the "LCR" lowpass filter by the mean of constrained evolution and with initial data given in previous subsection. The best result has been obtained at chromosome 17,308 of generations 62 $(20,000 \times 61+17,308=1,236,308$ individuals $)$, with 27 elements (without embryo-circuit), with the best fitness value 0.008084 . The schematic and the voltage response of the best circuit are shown on Fig.8. As could be seen we have got non-monotonic filter with the following features: the maximum absolute attenuation in passband is $0.0023 \mathrm{~dB}$, the maximum attenuation in stopband is $60 \mathrm{~dB}$.

We have set the rule that checks invalid circuits up to 5 nodes in the chain around new/mutated element whether they are floating, and the rule that checks up to 4 elements around new/mutated element whether they involved in the inductor/voltage source loop.

\section{Experiment B: Unconstrained evolution}

The purpose of this experiment is the unconstrained evolution of low-pass filter in comparison with previous constrained one. We use the same initial data given in subsection V-A. The best result has been obtained at chromosome 9,958 of generations 75 $(20,000 \times 74+9,958=1,857,453$ individuals $)$, with 28 elements before simplification, with the best fitness value 0.003916 , which is 2 times better than one got in constrained evolution (Experiment A). The schematic after simplification and the 
voltage response of the best circuit are shown on Fig.9.

As could be seen we have got non-monotonic filter consisting of 16 elements (without embryo) among which 1 is R-support and with the following features: the maximum absolute attenuation in the passband is $0.0043 \mathrm{~dB}$, the maximum attenuation in the stopband is $-69 \mathrm{~dB}$.
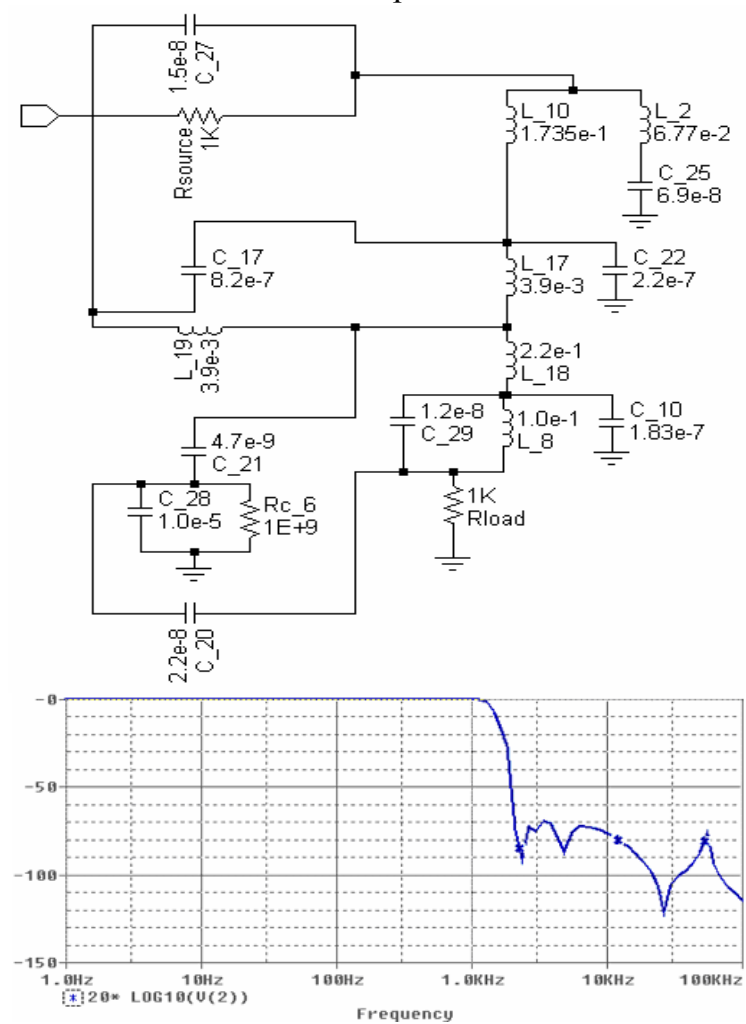

Fig.9. The schematic after simplification and the voltage response of the best low-pass filter evolved at Experiment B.

\section{THE COMPARISON OF CONSTRAINED AND UNCONSTRAINED EVOLUTIONS}

The experimental results show that exploiting the circuitstructure-checking rules in Experiment A still allow significant amount of mistaken circuits to be sent to simulation software. For example, in our case the rules still allowed about up to $15 \%$ of error circuits in average to be generated, which reduces the effective population size. In contrast, during running the Experiment $\mathrm{B}$, the amount of invalid graphs among all randomly generated ones did not

TABLE 2. COMPARISON TABLE OF FILTER AND EVOLUTION CHARACTERISTICS AMONG WORKS PUBLISHED BEFORE AND PRESENT. N/A MEANS THAT THE DATA ARE NOT AVAILABLE.

\begin{tabular}{|c|c|c|c|c|c|c|c|c|c|c|}
\hline & $\begin{array}{l}\text { Ideal } \\
\text { filter }\end{array}$ & $\begin{array}{c}\text { 10order } \\
\text { Chebyshev } \\
\text { filter }[15]\end{array}$ & $\begin{array}{c}\text { Koza } 1 \text { et } \\
\text { al, [2] } \\
\text { elliptic } \\
\end{array}$ & $\begin{array}{c}\text { Koza } 2 \text { et } \\
\text { al, [2] } \\
\text { ladder }\end{array}$ & $\begin{array}{c}\text { Koza } 3 \text { et } \\
\text { al, [2] } \\
\text { bridge-T }\end{array}$ & $\begin{array}{c}\text { Lohn } \\
\text { et al, } \\
{[3]} \\
\end{array}$ & $\begin{array}{l}\text { Goh, et } \\
\text { al [5] }\end{array}$ & $\begin{array}{l}\text { Zebulum } \\
\text { et al [4] }\end{array}$ & $\begin{array}{c}\text { Constrained } \\
\text { evolution }\end{array}$ & $\begin{array}{c}\text { Unconstrained } \\
\text { evolution }\end{array}$ \\
\hline \multicolumn{11}{|c|}{ Filter Characteristics } \\
\hline Pass band, $\mathrm{V}$ & 1 & 1 & 1 & 1 & 1 & 1 & 1 & 2 & 1 & 1 \\
\hline Stop band, V & 0 & 0 & 0 & 0 & 0 & 0 & 0 & 0 & 0 & 0 \\
\hline Transition band, $\mathrm{KHz}$ & 0 & 1 & 1 & 1 & 1 & 1 & 1 & 1 & 1 & 1 \\
\hline $\begin{array}{l}\text { Maximum absolute attenu- } \\
\text { ation in the pass-band, } \mathrm{dB}\end{array}$ & 0 & 0.035 & 0.179 & 0.0175 & 0.137 & 0.0144 & 0.042 & 0.188 & 0.0023 & 0.0043 \\
\hline$\%$ of improvement & - & $714 \%$ & $4063 \%$ & $307 \%$ & $3086 \%$ & $235 \%$ & $877 \%$ & $4272 \%$ & $-47 \%$ & - \\
\hline $\begin{array}{l}\text { Maximum attenuation in the } \\
\text { stop band, } d B\end{array}$ & $-\infty$ & -83 & -72 & -61 & -60 & -59 & -34 & -24 & -60 & -69 \\
\hline$\%$ of improvement & - & $-20 \%$ & $-4 \%$ & $12 \%$ & $13 \%$ & $14 \%$ & $51 \%$ & $65 \%$ & $13 \%$ & - \\
\hline
\end{tabular}




\begin{tabular}{|c|c|c|c|c|c|c|c|c|c|c|}
\hline Fitness value & & 0.0259 & 0.0805 & 0.0071 & 0.0502 & 0.0134 & 0.1858 & 585.7665 & 0.0081 & 0.0039 \\
\hline$\%$ of improvement & & $564 \%$ & $1964 \%$ & $82 \%$ & $1187 \%$ & $244 \%$ & $4664 \%$ & $1.5 \mathrm{e}+8 \%$ & $108 \%$ & - \\
\hline No. Elements & - & 10 & 25 & 14 & 15 & 24 & 12 & 10 & 27 & 16 \\
\hline No. Individuals & & - & $\mathrm{N} / \mathrm{A}$ & $2,048,000$ & N/A & 997,000 & 20,200 & 320,000 & $1,236,308$ & $1,489,958$ \\
\hline Circuit simulator & & & & MicroSim & & SPICE & MicroSim & SMASH & \multicolumn{2}{|c|}{ OrCAD } \\
\hline
\end{tabular}

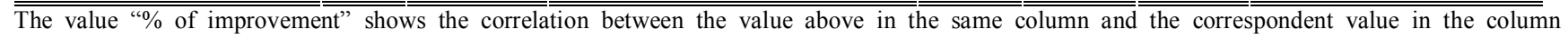
"Unconstrained evolution".

proposed technique is based on Evolutionary Strategy in combination with oscillating length genotype sweeping strategy. The unconstrained evolution demonstrated the superior behavior over the constrained one. The best fitness value obtained previously has been improved by $82 \%$. Thus, the instinctive wish to reduce the potential solution space for evolutionary search, by which usually the circuit-structurechecking rules are justified, is not always the best strategic maneuver for reducing the search time and obtaining good results.

The paper has shown one of the first successful attempts of application of Evolutionary Strategy towards analogue circuit design.

The oscillating length genotype sweeping strategy with capability of evolution to focus on limited oscillating genotype lengths has been developed. The results of experiment agree with ones in [4], where OLG strategy is the best for low-pass filter design.

The further analysis of results discovers the implicit tendency of evolution to minimize the usage of resistance and R-support elements such that the final solution could not contain them at all.

\section{REFERENCES}

[1] A. Thompson, "Hardware Evolution: Automatic Design of Electronic Circuits in Reconfigurable Hardware by Artificial Evolution", $P h D$ thesis, University of Sussex, Brighton, Sussex, England, 1996.

[2] J. R Koza, F.H. Bennett III, D. Andre, M.A. Keane, and F. Dunlap, "Automated Synthesis of Analog Electrical Circuit by Means of Genetic Programming", IEEE Trans. Evolutionary Computation, Vol.1, No.2, 1997.

[3] J. D. Lohn, S.P.Colombano, "Automated Analog Circuit Synthesis using a Linear Representation”, Proc. of the 2nd Int'l Conf. on

Evolvable Systems: From Biology to Hardware, Springer-Verlag, Berlin, 1998, pp.125- 133.

[4] R. S. Zebulum, M.A. Pacheco, M. Vellasco, "Comparison of different evolutionary methodologies applied to electronic filter design", IEEE Trans. on Conf. on Evolutionary Computation, Piscataway, NJ: IEEE Press, 1998, pp. 434-439.

[5] C. Goh, Y. Li, "GA automated design and synthesis of analog circuits with practical constraints", Proc. of the 2001 Congress on Evolutionary Computation, Vol. 1, pp. 170-177, 2001.

[6] S. Ando, H. Iba, Analog Circuit Design with a Variable Length Chromosome, Congress on Evolutionary Computation, pp.994-100, IEEE Press, 2000.

[7] J. B. Grimbleby, "Hybrid genetic algorithms for analogue network synthesis", Congress on Evolutionary Computing, Washington USA, pp. 1781-1787, 1999.

[8] T.R. Dastidar, P.P. Chakrabarti, P. Ray, "A synthesis system for analog circuits based on evolutionary search and topological reuse", IEEE Trans. Evolutionary Computation, Volume 9, Issue 2, April 2005, pp. $211-224$

[9] T. Sripramong, C. Toumazou, "The invention of CMOS amplifiers using genetic programming and current-flow analysis", IEEE Trans. Computer-Aided Design of Integrated Circuits and Systems, Volume 21, Issue 11, Nov. 2002, pp.1237-1252

[10] R. Zebulum, A. Stoica, D. Keymeulen, "Experiments on the Evolution of Digital to Analog Converters", Proc. of the 2001 IEEE Aerospace Conference, March 10-17 2001, Big Sky, Montana, USA Manhattan Beach, CA ISBN: 0-78-3-6600-X. (Published in CD)

[11] J. Hu, X. Zhong, E. Goodman, "Openended Robust Design of Analog Filters Using Genetic Programming", Proc. of Genetic \& Evolutionary Computation Conference-2005 (GECCO-2005), Vol. 2, ACM Press, Washington, DC, pp. 1619-1626, June, 2005.

[12] T. Kuo, S.-H. Hwang, "Using disruptive selection to maintain diversity in genetic algorithms", Appl. Intel. 7, 1997, pp. 257-267.

[13] M. Brameier, "On Linear Genetic Programming", PhD thesis, University of Dortmund, Dortmund, Germany, February 2004.

[14] A. Thompson, "Artificial evolution in the physical world", in Gomi (Ed.) Evolutionary Robotics, AAI Books, 1997.

[15] LC filter design. Available: http://www-users.cs.york.ac.uk/ fisher/lcfilter/ 